\title{
Comparative safety evaluation of 7-valent and 13 -valent pneumococcal vaccines in routine paediatric vaccinations in four Italian regions, 2009 to 2011
}

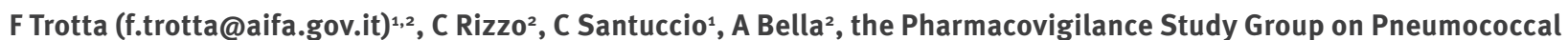
Vaccination in Children ${ }^{3}$

1. Pharmacovigilance Unit, Italian Medicines Agency, Rome, Italy

2. National Centre of Epidemiology, National Institute of Health, Rome, Italy

3. The members of the group are listed at the end of the article

Citation style for this article:

Trotta F, Rizzo C, Santuccio C, Bella A, the Pharmacovigilance Study Group on Pneumococcal Vaccination in Children. Comparative safety evaluation of 7-valent and 13-valent pneumococcal vaccines in routine paediatric vaccinations in four Italian regions, 2009 to 2011. Euro Surveill. 2015;20(7):pii=21041. Available online: http://www.eurosurveillance.org/ViewArticle.aspx?Articleld=21041

Article submitted on 21 January 2014 / published on 19 February 2015

This study was aimed at estimating the risk of all types of adverse events following immunisation $(A E F I)$, neurological events and convulsions following the co-administration of 13 -valent pneumococcal polysaccharide conjugate vaccine $\left(\mathrm{PCV}_{13}\right)$ with hexavalent vaccine. Paediatric spontaneous reports and exposure to vaccinations in four Italian regions were available. The estimated incidence rate ratio (IRR) for AEFI following co-administration of hexavalent vaccine with either $\mathrm{PCV}_{13}$ or $\mathrm{PCV}_{7}$ was 1.08 (95\% confidence interval (CI): 0.91-1.28); the IRR for, respectively, neurological events and convulsion following co-administration of $\mathrm{PCV}_{13}$ with hexavalent vaccine were $\mathbf{1 . 2 7}$ (95\% Cl: $0.85-1.89)$ and 1.43 (95\% Cl: 0.70-2.91). Co-administration of $\mathrm{PCV}_{13}$ with hexavalent vaccine had a protective effect against AEFI (IRR $=0.59 ; 95 \%$ $\mathrm{Cl}: 0.49-0.72)$. This protective effect was not observed for neurological events or convulsions following coadministration of $\mathrm{PCV}_{13}$ with hexavalent vaccine compared with single administration (IRR $=1.44 ; 95 \% \mathrm{Cl}$ : $0.77-2.67$ and IRR $=1.46 ; 95 \% \mathrm{Cl}: 0.50-4.25$, respectively). We observed a trend of increased risk of neurological events or convulsions following PCV13 used in routine practice. Analysis of spontaneously reported data is a quick method to estimate associations between vaccines and less common adverse events. Given methodological limitations these findings cannot be conclusive and require further investigations.

\section{Introduction}

The 13-valent pneumococcal polysaccharide conjugate vaccine $\left(P C V_{13}\right)$ was introduced in Italy in mid-2010, fully replacing the use of 7 -valent vaccine $\left(P C V_{7}\right)$ [1]. The $\mathrm{PCV}_{13}$ provides protection against an additional six serotypes $(1,5,7 \mathrm{~F}, 3,6 \mathrm{~A}, 19 \mathrm{~A})$ not included in $\mathrm{PCV}_{7}$.

Since 2005, pneumococcal vaccination has been recommended in the Italian national immunisation programme (NIP), but only for risk groups (e.g. asplenia, immunocompromised subjects, patients with chronic diseases) $[2,3]$. However, it was in the following years increasingly offered also to other target groups, dependent on the vaccination policies in the individual regions [2]. Since 2008 , pneumococcal vaccination has been included in the NIP free of charge for all newborns; it was administered as a three-dose schedule (during the first year of life) concomitantly with the hexavalent vaccine against diphtheria (D), tetanus ( $T$ ), acellular pertussis (aP), Haemophilus influenzae type $b$ (Hib), hepatitis B virus (HBV) and inactivated poliovirus (IPV) $[4,5]$. Thus, in routine practice, PCV and the hexavalent vaccine were usually administered concomitantly to children in a single vaccination session.

Clinical trials evaluating the effect of co-administration of PCV13 with the hexavalent vaccine showed a comparable safety and immunogenicity profile as those evaluating co-administration of $\mathrm{PCV}_{7}$ and the hexavalent vaccines when given in routine practice; among systemic adverse events, fever was more common in subjects vaccinated with $\mathrm{PCV}_{13}$ compared with $\mathrm{PCV}_{7}$ $[6,7]$. Like other vaccines, PCV can provoke fever, which could trigger a febrile seizure [7,9]. However, those clinical trials were not sized to detect less common or specific adverse events following immunisation (AEFI), and only involved a selected paediatric population (children with risk conditions were excluded). Therefore, post-marketing surveillance remains essential. The routine monitoring of spontaneous reports collected by the Italian Pharmacovigilance Network (IPN) in the first year after introduction of $\mathrm{PCV}_{13}$ in Italy showed a slightly higher frequency of serious AEFI with PCV13 than with $\mathrm{PCV}_{7}$; this trend was more evident for neurological events and when $\mathrm{PCV}_{13}$ was co-administered with the hexavalent vaccine [10]. This finding led us to 
Frequency of spontaneous reports of adverse events following vaccination, by seriousness and vaccine type administered alone or in co-administration, four regions in Italy, 2009 to $2011(\mathrm{n}=883)$

\begin{tabular}{|c|c|c|c|c|c|c|}
\hline & PCV7 alone & PCV13 alone & Hexavalent alone & $\begin{array}{c}\text { PCV7 and } \\
\text { hexavalent } \\
\text { co-administered }\end{array}$ & $\begin{array}{c}\text { PCV13 and } \\
\text { hexavalent } \\
\text { co-administered }\end{array}$ & \multirow[t]{2}{*}{ Total } \\
\hline & $\begin{array}{c}2009+ \\
\mathrm{Q}_{1-Q_{2} 2010}\end{array}$ & $\begin{array}{c}Q_{3}-Q_{4} 2010 \\
+2011\end{array}$ & $2009-2011$ & $\begin{array}{c}2009+ \\
Q_{1}-Q_{2} 2010\end{array}$ & $\begin{array}{c}Q_{3}-Q_{4} 2010 \\
+2011\end{array}$ & \\
\hline & n (\%) & $n(\%)$ & n (\%) & n (\%) & $n(\%)$ & n (\%) \\
\hline \multicolumn{7}{|l|}{ All AEFI $(n=883)$} \\
\hline Serious & $4(9)$ & $6(13)$ & $14(5)$ & $27(12)$ & $56(18)$ & $107(12)$ \\
\hline Not serious & $40(91)$ & $40(87)$ & $238(93)$ & $201(86)$ & $247(81)$ & $766(87)$ \\
\hline Undefined & o (o) & o (o) & $4(2)$ & $4(2)$ & $2(1)$ & $10(1)$ \\
\hline Total & $44(100)$ & $46(100)$ & $256(100)$ & $232(100)$ & $305(100)$ & $883(100)$ \\
\hline \multicolumn{7}{|c|}{ Neurological events $(n=136)$} \\
\hline Serious & $3(50)$ & $2(67)$ & $9(36)$ & $15(38)$ & $39(63)$ & $68(50)$ \\
\hline Not serious & $3(50)$ & $1(33)$ & $15(60)$ & $23(57)$ & $23(37)$ & $65(48)$ \\
\hline Undefined & o (o) & o (o) & $1(4)$ & $2(5)$ & o (o) & $3(2)$ \\
\hline Total & $6(100)$ & $3(100)$ & $25(100)$ & $40(100)$ & $62(100)$ & $136(100)$ \\
\hline \multicolumn{7}{|c|}{ Convulsions, febrile and afebrile $(n=41)$} \\
\hline Serious & $1(100)$ & $1(100)$ & $3(50)$ & $8(67)$ & $19(90)$ & $32(78)$ \\
\hline Not serious & o (o) & o (o & $3(50)$ & 4(33) & $2(10)$ & $9(22)$ \\
\hline Undefined & o (o) & o (o) & o (o) & o (o) & o (o) & $\mathrm{o}(0)$ \\
\hline Total & $1(100)$ & $1(100)$ & $6(100)$ & $12(100)$ & $21(100)$ & $41(100)$ \\
\hline Administered doses (n) & 209,098 & 169,069 & 243,802 & 802,126 & 979,446 & Not applicable \\
\hline
\end{tabular}

AEFI: adverse events following immunisation; PCV: pneumococcal polysaccharide conjugate vaccine.

further investigate the safety of $\mathrm{PCV}_{13}$ in combination with the hexavalent vaccine.

The first objective of this study was to estimate the risk of all types of AEFI, neurological events, and convulsions following the co-administration of $\mathrm{PCV}_{13}$ with the hexavalent vaccine, using the co-administration of $\mathrm{PCV}_{7}$ with the hexavalent vaccine as a reference. The second objective was to compare the risk of all types of AEFI, neurological events, and convulsions following the co-administration of $\mathrm{PCV}_{13}$ with the hexavalent vaccine vs single administration of $\mathrm{PCV}_{13}$ and the hexavalent vaccine in different vaccine sessions.

\section{Methods}

\section{AEFI reports and administered doses}

We retrieved paediatric spontaneous reports to IPN after vaccination with PCV (7- and 13-valent) or the hexavalent vaccine occurring from 1 January 2009 and 31 December 2011. Data retrieval from IPN took place in mid-2012. No exclusion criteria for cases of AEFI were adopted. Details on the vaccinations received during the three-year period, such as the number of doses of $\mathrm{PCV}_{7}, \mathrm{PCV}_{13}$ and the hexavalent vaccine administered to the paediatric population ( $0-2$ yearsold) both as single or concomitant vaccination, were available from four Italian regions, Emilia-Romagna, Lombardy, Tuscany and Veneto, and pooled at regional level. These regions are situated in the north and in the centre of Italy. All our analyses were limited to these regions, covering $22.6 \%$ of the resident Italian population and representing $77.8 \%$ of the spontaneous reports collected in the IPN during 2011 [11]. The corresponding paediatric population consisted of more than 217,000 children per year [12]. The birth cohort of children in 2012 had the following distribution by region: 41,397 in Emilia-Romagna, 96,602 in Lombardy, 32,473 in Tuscany, 46,588 in Veneto. The vaccine coverage (as completed vaccine course) during 2011 for DTaP, Hib, HBV, and IPV (included in the hexavalent vaccine) was at least $95 \%$ in all regions involved [13].

Each report was identified through a unique anonymised code and it was not possible to directly identify the person. We used information on the vaccinee (age, sex), the event(s) (type, date of onset, seriousness and outcome), vaccine(s) administered (type, trade name and date of administration), region and local healthcare facility indicated on the reporting form for the adverse drug reaction (ADR).

We considered two 18-month time periods to estimate the AEFI incidence rates (IR) of PCV7 and $\mathrm{PCV}_{13}$ (alone or co-administered with the hexavalent vaccine), from 1 January 2009 to 30 June 2010 for PCV 7 and from 1 July 2010 to 31 December 2011 for PCV13. All participating regional pharmacovigilance centres were asked 
to reconfirm the information reported for each case to exclude duplicates, increase data completeness and identify possible misclassification between $\mathrm{PCV}_{7}$ and PCV13. In Italy, during the period of interest, a single hexavalent vaccine was available.

Spontaneous ADR reports were grouped in three different categories, i.e. all the AEFI reported, neurological events, and convulsions. Neurological events were identified through the analysis of the reported AEFI and the related preferred terms coded according to the standardised medical terminology developed by the International Conference of Harmonization (MedDRA) [14]. Only cases with at least one preferred term leading to the primary MedDRA system organ classification (SOC) 'nervous system disorders' were considered as neurological events [15]. In addition, all serious cases were further evaluated (on the basis of the preferred terms, verbatim and other information included in the ADR reporting form) to confirm their inclusion/exclusion from the neurological events analysis.

All neurological reports were then evaluated to identify cases of convulsions (both febrile and afebrile) according to a pre-defined case definition. Cases reporting terms such as 'seizure' or 'convulsion' or 'convulsion and fever' were classified as convulsion/febrile convulsion by default. Cases with less specific terms (e.g. 'tonic-clonic movements', 'hypertonia', 'oculogyric crisis') were classified as convulsion only when two terms indicating loss of consciousness and generalised motor manifestation appeared together in the same report; this is in accordance with current case definition guidelines [16].

\section{Statistical analysis}

IR of AEFI were calculated by dividing the number of the $A E F I$ reports by the number of administered vaccine doses (expressed per 100,000 doses). The administered doses in the group receiving single administrations of $\mathrm{PCV}_{13}$ and the hexavalent vaccine at different times were calculated as the sum of the administered doses of $\mathrm{PCV}_{13}$ alone and the hexavalent vaccine alone. Confidence intervals $(95 \% \mathrm{Cl})$ were calculated using the Poisson distribution. Incidence rate ratios (IRR) were estimated using univariate Poisson regression. IR and IRR were estimated for three AEFI groups: (i) all types of AEFI, (ii) neurological events, (iii) convulsions (febrile and afebrile). An attempt to stratify AEFI groups by seriousness of event was made. A standard pharmacovigilance definition for seriousness of cases was used:

"An adverse reaction corresponds to any untoward medical occurrence that at any dose results in death, is life-threatening, requires inpatient hospitalisation or prolongation of existing hospitalisation, results in persistent or significant disability or incapacity, is a congenital anomaly/birth defect [17]"
All events which did not meet the criteria for seriousness were thus considered as non-serious. Because seriousness was analysed as reported and was not modified during the analysis, we need to consider that misclassification may have occurred. This study was not intended to investigate risks by seriousness of events, and the stratified analyses should be viewed as a hypothesis generator only. Statistical analyses were carried out using STATA software version 11.2 (Stata Corporation, College Station, United States).

\section{Results}

\section{ADR reports and administered doses}

According to the NIP, administration of PCV (7- or 13-valent) together with the hexavalent vaccine was at least four times more frequent than single administration of PCV (7- or 13-valent) or the hexavalent vaccine (Table 1); the number of co-administered doses of PCV (7- or 13-valent) with the hexavalent vaccine was comparable.

Overall, 883 spontaneous reports of $\mathrm{AEFI}$ with $\mathrm{PCV}_{7}$, $\mathrm{PCV}_{13}$ or the hexavalent vaccine either as single or concomitant administration were retrieved in the IPN during the period 2009 to 2011 (Table 1); 107 reports were serious (12.1\%), including two deaths of which one occurred two days after vaccination in a child suffering from perinatal hypoxic ischaemic encephalopathy and the other was sudden infant death syndrome. At least one neurological event was reported in $15.4 \%$ of the reports (136 of 883); 68 (50.0\%) neurological events were serious. Of the 136 neurological events, $41(30.1 \%)$ were cases of convulsions and 32 of the 41 were reported as serious. In particular, we found that of 41 cases of convulsion, 26 were reported as febrile convulsion, while the remaining 15 cases were afebrile convulsions; given the small sample we considered convulsion (both febrile and afebrile) as a single category in the analyses.

The majority of reports $(n=537), 15.4 \%$ of which were serious $(n=83)$, occurred after co-administration of $\mathrm{PCV}_{7}$ or $\mathrm{PCV}_{13}$ with the hexavalent vaccine $(\mathrm{n}=232$ and $n=305$, respectively). The hexavalent vaccine alone was found to be administered in $256 \mathrm{AEFI}$ reports, $5.5 \%$ of them serious $(n=14)$, while we found only 90 reports following a single administration of $\mathrm{PCV}_{7}$ or $\mathrm{PCV}_{13}, 10$ of which were serious (four with $\mathrm{PCV}_{7}$ and six with P(V13).

Reports of any AEFI (serious and not serious) were equally frequent for any of the three vaccines, whether administered alone or concomitantly. In contrast, serious AEFI, neurological events and convulsions were observed more frequently when $\mathrm{PCV}_{13}$ and the hexavalent vaccine were administered together.

Overall, the demographic characteristics of children experiencing an AEFI were comparable across vaccine groups (Table 2). However, according to the vaccine 


\section{TABLE 2}

Spontaneous reports of adverse events following vaccination, by sex, age group, and time to onset, four regions in Italy, 2009 to $2011(\mathrm{n}=883)$

\begin{tabular}{|c|c|c|c|c|c|}
\hline & PCV7 alone & PCV13 alone & Hexavalent alone & $\begin{array}{c}\text { PCV7 and } \\
\text { hexavalent } \\
\text { co-administered }\end{array}$ & $\begin{array}{c}\text { PCV13 and } \\
\text { hexavalent } \\
\text { co-administered }\end{array}$ \\
\hline & $\begin{array}{c}2009+ \\
\mathrm{Q}_{1-Q_{2} 2010}\end{array}$ & $\begin{array}{l}Q_{3}-Q_{4} 2010 \\
+2011\end{array}$ & $2009-2011$ & $\begin{array}{c}2009+ \\
Q_{1-Q 22010}\end{array}$ & $\begin{array}{c}Q_{3}-Q_{4} 2010 \\
+2011\end{array}$ \\
\hline & n (\%) & n (\%) & n (\%) & n (\%) & $\mathrm{n}(\%)$ \\
\hline \multicolumn{6}{|l|}{ Sex ${ }^{a}$} \\
\hline Male & $21(48)$ & $28(61)$ & $150(59)$ & $120(52)$ & $157(51)$ \\
\hline Female & $23(52)$ & $18(39)$ & $106(41)$ & $111(48)$ & $148(49)$ \\
\hline \multicolumn{6}{|l|}{ Age group ${ }^{a}$} \\
\hline$<6$ months & $6(14)$ & 4(9) & $138(54)$ & $158(68)$ & $216(71)$ \\
\hline 6-11 months & $8(19)$ & $5(11)$ & $68(27)$ & $38(16)$ & $54(18)$ \\
\hline $12-24$ months & $29(67)$ & $36(80)$ & 49 (19) & $36(16)$ & $35(11)$ \\
\hline \multicolumn{6}{|l|}{ All AEFI } \\
\hline \multicolumn{6}{|l|}{ Time to onset ${ }^{\mathrm{b}}$} \\
\hline o days & $24(56)$ & $28(62)$ & $180(70)$ & $190(82)$ & $248(82)$ \\
\hline 1 days & $12(28)$ & $13(29)$ & $54(21)$ & $29(13)$ & $34(11)$ \\
\hline$\geq 2$ days & $7(16)$ & $4(9)$ & $22(9)$ & $12(5)$ & $22(7)$ \\
\hline \multicolumn{6}{|l|}{ ADR outcome $\mathrm{e}^{\mathrm{b}}$} \\
\hline Resolved/improved & $30(68)$ & $43(94)$ & $217(85)$ & $212(91)$ & $263(69)$ \\
\hline Unresolved & o (o) & $1(2)$ & $1(0)$ & $5(2)$ & $9(2)$ \\
\hline Not available & $14(32)$ & $2(4)$ & $38(15)$ & $15(7)$ & 109 (29) \\
\hline \multicolumn{6}{|l|}{ Neurological events } \\
\hline \multicolumn{6}{|l|}{ Time to onset ${ }^{\mathrm{b}}$} \\
\hline o days & $5(83)$ & $3(100)$ & $21(84)$ & $30(77)$ & 49 (79) \\
\hline 1 days & 0 & 0 & $1(4)$ & $7(18)$ & $10(16)$ \\
\hline$\geq 2$ days & $1(17)$ & 0 & $3(12)$ & $2(5)$ & $3(5)$ \\
\hline \multicolumn{6}{|l|}{ ADR outcome ${ }^{b}$} \\
\hline Resolved/improved & $4(67)$ & $3(100)$ & $22(88)$ & $38(94)$ & $52(84)$ \\
\hline Still not resolved & 0 & 0 & 0 & $1(3)$ & $1(2)$ \\
\hline Not available & $2(33)$ & 0 & $3(12)$ & $1(3)$ & $9(14)$ \\
\hline \multicolumn{6}{|c|}{ Convulsions (febrile and afebrile) } \\
\hline \multicolumn{6}{|c|}{ Time to onset ${ }^{\mathrm{b}}$} \\
\hline o days & $1(100)$ & $1(100)$ & $4(66)$ & $8(66)$ & $15(71)$ \\
\hline 1 days & 0 & 0 & $1(17)$ & $2(17)$ & $5(24)$ \\
\hline$\geq 2$ days & 0 & 0 & $1(17)$ & $2(17)$ & $1(5)$ \\
\hline \multicolumn{6}{|l|}{ ADR outcome ${ }^{b}$} \\
\hline Resolved/improved & $1(100)$ & $1(100)$ & $4(67)$ & $10(84)$ & $17(81)$ \\
\hline Still not resolved & 0 & 0 & 0 & $1(8)$ & 0 \\
\hline Not available & 0 & 0 & $2(33)$ & $1(8)$ & $4(19)$ \\
\hline
\end{tabular}

ADR: adverse drug reaction; AEFI: adverse events following immunisation; ICSR: individual case safety report; PCV: pneumococcal polysaccharide conjugate vaccine.

a The sum of the variables sex and age group differs from the overall population enrolled due to missing information reported on the ICSR.

b The sum of the variables time to onset and ADR outcome differs from the overall population enrolled since such information is referred to the ADRs reported in each ICSR, and each ICSR may include more than one reaction.

c At the time when the report was filed. 
Comparison of incidence ratios between PCV7 vs PCV13 given in co-administration with the hexavalent vaccine, four regions in Italy, 2009 to $2011(\mathrm{n}=537)$

\begin{tabular}{|c|c|c|c|c|c|c|}
\hline \multirow{3}{*}{ Administered doses (n) } & \multicolumn{2}{|c|}{$\begin{array}{l}\text { PCV7 and hexavalent } \\
\text { Co-administered } \\
\text { 2009+Q1-Q2 } 2010\end{array}$} & \multicolumn{2}{|c|}{$\begin{array}{l}\text { PCV13 and hexavalent } \\
\text { C0-administered } \\
\text { Q3-Q4 2010+2011 }\end{array}$} & \multicolumn{2}{|c|}{$\begin{array}{l}\text { PCV13 and hexavalent } \\
\text { CO-administered } \\
\text { vs PCV7 and hexavalent } \\
\text { co-administered }\end{array}$} \\
\hline & & 802,126 & & 979,446 & & \\
\hline & $\mathrm{n}$ & IR $(95 \% \mathrm{Cl})$ & $\mathrm{n}$ & IR $(95 \% \mathrm{Cl})$ & IRR & $(95 \% \mathrm{Cl})$ \\
\hline \multicolumn{7}{|l|}{ All AEFI $(n=537)$} \\
\hline Serious & 27 & $3.37(2.22-4.90)$ & 56 & $5.72(4.32-7.42)$ & 1.70 & $(1.07-2.69)$ \\
\hline Not serious & 201 & $25.06(21.71-28.77)$ & 247 & $25.22(22.17-28.57)$ & 1.01 & $(0.83-1.21)$ \\
\hline Undefined & 4 & $0.49(0.13-1.28)$ & 2 & $0.20(0.02-0.74)$ & 0.41 & $(0.07-2.23)$ \\
\hline Total & 232 & $28.92(25.32-32.89)$ & 305 & $31.14(27.74-34.84)$ & 1.08 & $(0.91-1.28)$ \\
\hline \multicolumn{7}{|c|}{ Neurological events $(n=102)$} \\
\hline Serious & 15 & $1.87(1.05-3.08)$ & 39 & $3.98(2.83-5.44)$ & 2.13 & $(1.17-3.86)$ \\
\hline Not serious & 23 & $2.87(1.82-4.30)$ & 23 & $2.35(1.49-3.52)$ & 0.82 & $(0.46-1.46)$ \\
\hline Undefined & 2 & $0.25(0.03-0.90)$ & 0 & & Not applicable & \\
\hline Total & 40 & $4.99(3.56-6.79)$ & 62 & $6.33(4.85-8.11)$ & 1.27 & $(0.85-1.89)$ \\
\hline \multicolumn{7}{|c|}{ Convulsions, febrile and afebrile $(n=33)$} \\
\hline Serious & 8 & $1.00(0.43-1.96)$ & 19 & $1.94(1.17-3.03)$ & 1.94 & $(0.85-4.44)$ \\
\hline Not serious & 4 & $0.49(0.13-1.28)$ & 2 & $0.20(0.02-0.74)$ & 0.41 & $(0.07-2.23)$ \\
\hline Undefined & o & 0 & 0 & 0 & Not applicable & \\
\hline Total & 12 & $1.50(0.77-2.61)$ & 21 & $2.14(1.33-3.28)$ & 1.43 & $(0.70-2.91)$ \\
\hline
\end{tabular}

AEFI: adverse events following immunisation; CI: confidence interval; IR: incidence rate; PCV: pneumococcalpolysaccharide conjugate vaccine.

strategy adopted in Italy, children up to five completed months of age accounted for ca $70 \%$ of reports following co-administration of PCV (7- or 13-valent) with the hexavalent vaccine, while the majority of reports following single administration of $\mathrm{PCV}_{7}(67 \%)$ or $\mathrm{PCV}_{13}(80 \%)$ occurred in children that were at least 12 months-old.

On average, onset of the AEFI was on the same day as vaccine administration in $75.4 \%$ of the reports, ranging from a minimum of $55.8 \%$ for single $\mathrm{PCV}_{7}$ to a maximum of $82.2 \%$ for the co-administration of $\mathrm{PCV}_{7}$ with the hexavalent vaccine. The majority of the AEFI reported $(86.6 \%)$ resolved or improved. No difference in event onset or ADR outcome was found between the two types of co-administrations across different AEFI groups (Table 2 ).

\section{PCV7 or PCV13 co-administered with the}

hexavalent vaccine: AEFI incidence rates and incidence rate ratios

Overall, no differences in IR (for all AEFI, neurological events or convulsions) were observed following $\mathrm{PCV}_{7}$ co-administration or $\mathrm{PCV}_{13}$ co-administration (Table 3). The IRR for all AEFI following the co-administration of $\mathrm{PCV}_{13}$ with the hexavalent vaccine compared with the co-administration of $\mathrm{PCV}_{7}$ with the hexavalent vaccine was 1.08 (95\% Cl: 0.91-1.28). Similarly, the IRR for neurological events or convulsion following co-administration of $\mathrm{PCV}_{13}$ with the hexavalent vaccine were
1.27 (95\% Cl: $0.85-1.89$ ) and 1.43 (95\% Cl: $0.70-2.91$ ), respectively. None of the three comparisons were statistically significant. When taking into account the seriousness of reactions, the IRR for all types of AEFI and for neurological events indicated an increased risk reaching statistical significance, and a similar risk trend was observed for serious convulsions (Table 3 ).

\section{Co-administration of PCV13 with the}

hexavalent vaccine vs single administration of PCV13 and the hexavalent vaccine: AEFI incidence rates and incidence rate ratios The AEFI IR for the co-administration of $\mathrm{PCV}_{13}$ with the hexavalent vaccine were compared with those observed when PCV 13 and the hexavalent vaccine were administered in different vaccination sessions. Overall, a lower significant IR for all AEFI was observed following the co-administration of $\mathrm{PCV}_{13}$ and the hexavalent vaccine (Table 4 ). In case of neurological events and convulsions the IR did not differ between the two groups. When considering the IRR, co-administration of PCV13 with the hexavalent vaccine was also associated with a smaller number of all types of AEFI compared with the single administration of PCV13 and the hexavalent vaccine at different times (IRR: 0.59, 95\% $\mathrm{Cl}$ : 0.49-0.72) (Table 4). This protective effect was not observed for neurological events or convulsions $(I R R=1.44 ; 95 \% \mathrm{Cl}: 0.77-2.67$ and $I R R=1.46 ; 95 \%$ $\mathrm{Cl}: 0.50-4.25$, respectively). Taking into account the seriousness of events (serious AEFI overall, serious 
Comparison of incidence ratios of co-administration of PCV13 with the hexavalent vaccine vs separate administration in different vaccination sessions, four regions in Italy, 2009 to $2011(\mathrm{n}=448)$

\begin{tabular}{|c|c|c|c|c|c|c|}
\hline & \multicolumn{2}{|c|}{$\begin{array}{c}\text { PCV13 and hexavalent administered } \\
\text { in different vaccination sessionsa } \\
Q_{3}-Q_{4} 2010+2011\end{array}$} & \multicolumn{2}{|c|}{$\begin{array}{l}\text { PCV13 and hexavalent } \\
\text { Co-administered } \\
\text { Q3-Q4 2010+2011 }\end{array}$} & \multicolumn{2}{|c|}{$\begin{array}{c}\text { PCV13 and hexavalent } \\
\text { co-administered in the same session } \\
\text { vs single administration in different } \\
\text { sessions }\end{array}$} \\
\hline \multirow[t]{2}{*}{ Administered doses (n) } & \multicolumn{2}{|r|}{272,494} & \multicolumn{2}{|r|}{979,446} & \multicolumn{2}{|c|}{ Not applicable } \\
\hline & $\mathrm{n}$ & IR $(95 \% \mathrm{Cl})$ & $\mathrm{n}$ & $\mathrm{IR}(95 \% \mathrm{CI})$ & IRR & $(95 \% \mathrm{Cl})$ \\
\hline \multicolumn{7}{|l|}{ All AEFI $(n=448)$} \\
\hline Serious & 9 & $3.30(1.51-6.27)$ & 56 & $5.72(4.32-7.42)$ & 1.73 & $(0.86-3.50)$ \\
\hline Not serious & 133 & $48.81(48.87-57.84)$ & 247 & $25.22(22.17-28.57)$ & 0.52 & $(0.42-0.64)$ \\
\hline Undefined & 1 & $0.37(0.005-2.04)$ & 2 & $0.20(0.02-0.74)$ & 0.56 & $(0.05-6.14)$ \\
\hline Total & 143 & $52.48(44.23-61.82)$ & 305 & $31.14(27.74-34.84)$ & 0.59 & $(0.49-0.72)$ \\
\hline \multicolumn{7}{|c|}{ Neurological events $(n=74)$} \\
\hline Serious & 5 & $1.83(0.59-4.28)$ & 39 & $3.98(2.83-5.44)$ & 2.17 & $(0.85-5.50)$ \\
\hline Not serious & 7 & $2.57(1.03-5.29)$ & 23 & $2.35(1.49-3.52)$ & 0.91 & $(0.39-2.13)$ \\
\hline Undefined & 0 & 0 & 0 & 0 & Not applicable & Not applicable \\
\hline Total & 12 & $4.40(2.27-7.69)$ & 62 & $6.33(4.85-8.11)$ & 1.44 & $(0.77-2.67)$ \\
\hline \multicolumn{7}{|c|}{ Convulsions, febrile and afebrile $(n=25)$} \\
\hline Serious & 2 & $0.73(0.08-2.65)$ & 19 & $1.94(1.17-3.03)$ & 2.64 & $(0.61-11.35)$ \\
\hline Not serious & 2 & $0.73(0.08-2.65)$ & 2 & $0.20(0.02-0.74)$ & 0.28 & $(0.04-1.97)$ \\
\hline Undefined & 0 & 0 & 0 & 0 & Not applicable & Not applicable \\
\hline Total & 4 & $1.47(0.39-3.76)$ & 21 & $2.14(1.33-3.28)$ & 1.46 & $(0.50-4.25)$ \\
\hline
\end{tabular}

ADR: adverse drug reaction; $A E F I$ : adverse events following immunisation; $\mathrm{CI}$ : confidence interval; IR: incidence rate; IRR: incidence rate ratio;

PCV: pneumococcal polysaccharide conjugate vaccine.

a The events following vaccine administration in different vaccination sessions were obtained as the sum of the number of ADR following single PCV13 (Q3-Q4 2010 and 2011) and the number of ADRs following a single hexavalent vaccine dose (Q3-Q4 2010 and 2011).

neurological events and serious convulsions), the IRR showed a trend of increased risk associated with coadministration, although statistical significance was not reached and $\mathrm{Cl}$ were wide.

\section{Discussion}

In this study, we found a trend of a slightly increased risk for neurological events or convulsion after coadministration of $\mathrm{PCV}_{13}$ with the hexavalent vaccine when compared with the co-administration of $\mathrm{PCV}_{7}$ with the hexavalent vaccine. No increased risk emerged when the comparison concerns all types of AEFI. Two factors could be responsible for this observation. The first is the Weber effect, i.e. the increased attention paid by the healthcare professionals (HCPs) and the public to the launch of the new product (PCV13) in mid2010 replacing the old product $\left(\mathrm{PCV}_{7}\right)$ which may have led to an increased incidence of reported cases. The second factor is an overall increased vaccine reporting trend over the years in Italy, fuelled by the launch of several active pharmacovigilance projects [18].

The second relevant finding of this study regards the comparison between different vaccination strategies with $\mathrm{PCV}_{13}$ and the hexavalent vaccine (i.e. whether co-administered or not). Co-administration of these two vaccines showed a slightly increased risk trend for neurological events or convulsion. However, when considering all AEFI, co-administration had a protective effect compared with single administration. Thus, the comparison between the two vaccination strategies seemed to favour co-administration, which is the current immunisation practice in Italy), over single administration. It should be pointed out that the protective effect of co-administration on all AEFI may have been influenced by the fact that the probability for an AEFI to be counted twice is higher when $\mathrm{PCV}_{13}$ and the hexavalent vaccine are given on different days: one AEFI may occur with PCV13 on one day and another AEFI may occur with the hexavalent vaccine on another day.

Only two studies have been published on the postmarketing surveillance of PCV, both based on data from the United States (US) and none aimed at evaluating PCV given in co-administration $[19,20]$. The first was a descriptive study by Wise et al., presenting ADR reports following $\mathrm{PCV}_{7}$ [19]; the second article by Tseng et al. was based on active surveillance data and investigated the association between pre-specified events (including febrile seizure) with $\mathrm{PCV}_{13}$ or $\mathrm{PCV}_{7}$; it did not find any signal of an increased risk for febrile convulsion [20]. However, this study did not report the $\mathrm{Cl}$ for risk estimates of febrile seizure and did not consider the effect of co-administration of PCV with other vaccines or other vaccination strategies. Of note, Tseng et al. discussed an ancillary analysis in children receiving 
PCV13 and influenza vaccine concomitantly; they found a risk trend for febrile seizure 1.35 times higher (95\% $\mathrm{Cl}$ : 0.93-2.00) than the risk after receiving $\mathrm{PCV}_{7}$ and influenza vaccine concomitantly [20].

A study based on active surveillance data was conducted in the US to investigate a signal of febrile seizures in young children after receipt of trivalent inactivated influenza vaccine (TIV) during the 2010/11 season. This study showed a risk of febrile seizure when TIV was co-administered with PCV13 that was higher than after single administration of TIV or PCV13 [21]. These findings were validated by an observational study conducted in the US which used prospective data collection and showed a higher risk of fever when TIV and PCV13 were co-administered than when either of these vaccines was administered without the other [22]. Increasing evidence suggests that co-administration and type of vaccine co-administered could play a role in the occurrence of an event such as convulsions following PCV. Overall, the results of our study are coherent with available literature data [19-22].

A strength of our study is that it used spontaneous reports from the general population exposed to vaccines without pre-defined exclusion/inclusion criteria. Moreover, our setting involved the whole paediatric population undergoing routine immunisation practice in the four regions included in our analysis. As these regions represent about a quarter of the country's total population, we consider our results to be representative for Italy as a whole.

The quality of spontaneous reporting data in Italy is high since an evaluation of each single case and validation of each report by a trained HCP is required [23]. Furthermore, all cases reporting neurological events were reviewed, and a predefined criterion to identify convulsions was applied.

The main limitation of this study resides in the nature of spontaneous reporting data. Although they allow the detection of less common events such as convulsions, they may contain partial information reported in a narrative way and collected heterogeneously. Moreover, the percentage of under-reporting could be significant, leading to a systematic under-estimation of cases; this would lead to decreased power of the study in detecting differences in the risks of AEFI, ultimately causing a lack of statistical significance and a wider $\mathrm{Cl}$. The higher risk estimates for serious cases can be expected to be affected by this methodological limitation. Seriousness has been analysed as indicated on the reports, not modified during the analysis, and could have introduced misclassification; the analyses by seriousness should thus be taken only as hypothesis-generating. Indeed, for a better interpretation of the study findings, it is advisable to refer to the overall risks within each ADR category. No risk adjustments for factors representing potential confounders (underlying diseases, age, sex) was feasible since this information was not available at individual level for the subjects undergoing vaccination (only administered doses pooled at regional level were retrieved); however, age, sex, time of onset and ADR outcome can be considered to be balanced among the spontaneous reports in each vaccine group, and the influence of these covariates on the risk estimates could be residual. The lack of anamnesis for neurological conditions both in the $A E F I$ reports and in each vaccinee's record is important missing information. Finally, we were not able to carry out any analyses by received dose since this information is not systematically included on the spontaneous report forms.

Even though safety surveillance based on spontaneously reported data is not intended to provide evidence on causality, it is a useful method to rapidly quantify associations between vaccines and less common adverse events. Moreover, preliminary findings based on surveillance data could help in designing further investigations to deliver more robust evidence.

\section{Conclusion}

Our analysis showed a trend of a slightly increased risk of neurological events or convulsions following vaccination with $\mathrm{PCV}_{13}$ compared with $\mathrm{PCV}_{7}$ when both were used in routine vaccination practice with the hexavalent vaccine. Similarly, we found an increased risk of neurological events or convulsions (although not reaching statistical significance) when $\mathrm{PCV}_{13}$ was co-administered with the hexavalent vaccine compared with single administration of both vaccines at different times. Given the limitations highlighted, our findings cannot be considered conclusive. Moreover, it should be underlined that such risks should be viewed in the context of the overall benefit of both vaccines.

While we continue monitoring reports of less common and potentially serious AEFI, further research should be conducted using different data sources that also account for dosing schedule, subjects' characteristics and co-morbidities. This study indicates that the evaluation of co-administration of PCV with other vaccines during a single session is a relevant issue for public health research. Our findings may also contribute to pooled estimates together with those of similar investigations.

\section{Acknowledgements}

Only public employees of the national or regional health authorities were involved in conceiving, planning, and conducting the study; no additional funding was received.

Disclaimer: The opinions expressed in this article do not necessarily reflects the view of the authors' institutions.

\section{Conflict of interest}

None declared. 


\section{Authors' contributions}

All authors conceived the study; FT, CR, CS, AB designed the study and analysed the data; FT, CR, CS, AB wrote the manuscript. All authors contributed to the discussion and reviewed the manuscript. All authors saw, commented upon and approved the final version of the paper.

\section{Members of the Pharmacovigilance Study Group on Pneumococcal Vaccination in Children}

Francesco Trotta (Pharmacovigilance Unit, Italian Medicines Agency and National Centre of Epidemiology, National Institute of Health, Rome); Carmela Santuccio, PatriziaFelicetti (Pharmacovigilance Unit, Italian Medicines Agency, Rome); Antonino Bella, Caterina Rizzo (National Centre of Epidemiology, National Institute of Health, Rome); Valentino Conti, Giuseppe Monaco (The Lombardy Region); Francesca Russo, Giovanna Zanoni(The Veneto Region); LoredanaOsbello, Maria GraziaPascucci (The Emilia Romagna Region); Maria Parrilli, Marco Rossi (The Tuscany Region).

\section{References}

1. Indicazioni in merito alla somministrazione del vaccino antipneumococcico Prevenar 13 in età pediatrica. [Recommendations on the administration of PCV 13 in the paediatric population]. Rome: Ministry of Health. 27 May 2010.

2. Piano nazionale vaccini $2005-2007$. [National immunization schedules 2005-2007]. Rome: Ministry of Health. 31 Dec 2005. Italian. Available from: http://www.salute.gov.it/portale/ documentazione/p6_2_2_1.jsp?lingua=italiano\&id=543

3. Vaccinazione antipneumococcica in età pediatrica. [Antipneumococcal vaccination in paediatric age]. Rome: Ministry of Health. 19 Nov 2001. Italian. Available from: http:// www.salute.gov.it/imgs/C_17_normativa_78_allegato.pdf

4. Alfonsi V, D’Ancona F, Giambi C, Nacca G, Rota MC, Regional Coordinators for Infectious Diseases and Vaccinations. Current immunization policies for pneumococcal, meningococcal $C$, varicella and rotavirus vaccinations in Italy. Health Policy. 2011;103(2-3):176-83. http://dx.doi.org/10.1016/j. healthpol.2011.10.002 PMID:22030308

5. Piano nazionale prevenzione vaccinale 2012-1014. [National immunisation schedules 2012-2014]. Rome: Ministry of Health 5 April 2012 [Accessed: 14 Jan 2014]. Italian. Available from: http://www.salute.gov.it/imgs/C_17_pubblicazioni_1721_ allegato.pdf

6. Esposito S, Tansey S, Thompson A, Razmpour A, Liang J, Jones TR, et al .Safety and immunogenicity of a 13-valent pneumococcal conjugate vaccine compared to those of a 7 -valent pneumococcal conjugate vaccine given as a threedose series with routine vaccines in healthy infants and toddlers. Clin Vaccine Immunol. 2010;17(6):1017-26. http:// dx.doi.org/10.1128/CVI.00062-10 PMID:20427630

7. Kieninger DM, Kueper K, Steul K, Juergens C, Ahlers N, Baker $\mathrm{S}$, et al. Safety, tolerability, and immunologic noninferiority of a 13-valent pneumococcal conjugate vaccine compared to a 7-valent pneumococcal conjugate vaccine given with routine pediatric vaccinations in Germany. Vaccine. 2010;28(25):4192203. http://dx.doi.org/10.1016/j.vaccine.2010.04.008 PMID:20417262

8. Frequently Asked Questions about Febrile Seizures Following Childhood Vaccinations. Atlanta: Centers for Disease Control and Prevention. [Accessed: 14 Jan 2014]. Available from: http:// www.cdc.gov/vaccinesafety/Concerns/FebrileSeizures.html

9. Cendes F, Sankar R. Vaccinations and febrile seizures. Epilepsia.2011;52(Suppl 3):23-5. http://dx.doi.org/10.1111/ j.1528-1167.2011.03032.x PMID:21542842

10. Santuccio C, Menniti-Ippolito F, Tartaglia L, Trotta F, Morlino D, Moretti U. Spontaneous Reporting of Adverse Reactions to Pneumococcal Vaccination in Children. Drug Saf. 2011;34(10):924-5.

11. Rapporto sulla sorveglianza postmarketing dei vaccini in Italia,2011. [Report on the post-marketing surveillance of vaccines in Italy,2011]. Rome: Agenzia Italiana del Farmaco (AIFA). [Accessed: 14 Jan 2014]. Italian. Available from: http:// www.agenziafarmaco.gov.it/sites/default/files/Copertina $\% 20$ +\%20Rapporto\%20vaccini\%202011_0.pdf
12. Resident population in Italy. Rome: The National Institute for Statistics. [Accessed: 21 Sep 2014]. Italian. Available from: http://demo.istat.it/index.html

13. Coperture vaccinali. [Vaccine coverage]. Rome: Ministry of Health. 4 January 2008. [Accessed: 21 Sep 2014]. Italian. Available from: http://www.salute.gov.it/imgs/C_17_ pagineAree_811_listaFile_itemName_15_file.pdf

14. Multidisciplinary Guidelines.M1 MedDRA Terminology. Geneva: The International Conference on Harmonization of Technical Requirements for Registration of Pharmaceuticals for Human Use (ICH). [Accessed: 14 Jan 2014]. Available from:http:// www.ich.org/products/guidelines/multidisciplinary/article/ multidisciplinary-guidelines.html

15. Introductory guide MedDRA Version 15.1. Geneva: The International Conference on Harmonization of Technical Requirements for Registration of Pharmaceuticals for Human Use (ICH). Sep2012. Available from:http://www.meddra.org/ sites/default/files/guidance/file/intguide_15_1_English_o.pdf

16. Bonhoeffer J, Menkes J, Gold MS, de Souza-Brito G, Fisher MC, Halsey N, et al. Generalized convulsive seizure as an adverse event following immunization: case definition and guidelines for data collection, analysis, and presentation. Vaccine. 2004;22(5-6):557-62. http://dx.doi.org/10.1016/j. vaccine.2003.09.008 PMID:14741144

17. European Directive. Directive $2001 / 83 / E C$ of the European Parliament and of the Council of 6 November 2001 on the Community code relating to medicinal products for human use. Official Journal of the European Union (OJ) L 311, 2001. Available from: http://www.echamp.eu/fileadmin/user upload/Regulation/Directive_2001-82-EC__-_Consolidated Version_.pdf

18. Trotta F, Alessandro A, Tartaglia L. Rapporto sul programma di farmacovigilanza attiva finanziato attraverso i fondi regionali disponibili per gli anni 2008 e 2009 . [Report on the active pharmacovigilance programme funded with regional funds available during fiscal year 2008 and 2009]. Rome: Agenzia Italiana del Farmaco, 2013. [Accessed: 21 Nov 2013]. Italian. Available from: http://www.agenziafarmaco.gov.it/sites/ default/files/AIFA_Rapporto\%20Fondi_FV.pdf

19. Wise RP, Iskander J, Pratt RD, Campbell S, Ball R, Pless RP, et al. Post licensure safety surveillance for 7 -valent pneumococcal conjugate vaccine.JAMA.2004;292(14):1702-10. http://dx.doi. org/10.1001/jama.292.14.1702 PMID:15479935

20. Tseng HF, Sy LS, Liul L, Qian L, Marcy SM, Weintraub E, et al. Post licensure surveillance for pre-specified adverse events following the 13-valent pneumococcal conjugate vaccine in children. Vaccine. 2013:31(22):2578-83. http://dx.doi. org/10.1016/j.vaccine.2013.03.040 PMID:23579258

21. Tse A, Tseng HF, Greene SK, Vellozzi C, Lee GM, VSD Rapid Cycle Analysis Influenza Working Group. Signal identification and evaluation for risk of febrile seizures in children following trivalent inactivated influenza vaccine in the Vaccine Safety Datalink Project, 2010-2011. Vaccine. 2012;30(11):202431. http://dx.doi.org/10.1016/j.vaccine.2012.01.027 PMID:22361304

22. Stockwell MS, Broder K, LaRussa P, Lewis P, Fernandez $\mathrm{N}$, Sharma $\mathrm{D}$, et al. Risk of fever after pediatric trivalent inactivated influenza vaccine and 13 -valent pneumococcal conjugate vaccine. JAMA Pediatr. 2014;168(3):211-9. http://dx.doi.org/10.1001/jamapediatrics.2013.4469 PMID:24395025</jrn>

23. Bergvall T, Noren GN, Lindquist M. Great case reports, where do they come from? Drug Saf. 2013;36(9):858. 